\title{
The mechanism through which octreotide inhibits hepatic stellate cell activity
}

\author{
JINGJING WANG $^{1 *}$, LIN WANG ${ }^{2,3^{*}}$, GUANHUA SONG ${ }^{4}$ and BO HAN ${ }^{2}$ \\ ${ }^{1}$ Clinical Test Department of Shandong Qianfoshan Hospital, Jinan, Shandong 250014; ${ }^{2}$ Department of Pathology, \\ Shandong University Medical School, Jinan, Shandong 250012; ${ }^{3}$ Research Center for Medicinal Biotechnology, \\ Shandong Academy of Medicinal Sciences, Jinan 250062; ${ }^{4}$ Institute of Basic Medicine, \\ Shandong Academy of Medical Sciences, Jinan, Shandong 250117, P.R. China
}

Received October 9, 2012; Accepted February 13, 2013

DOI: $10.3892 / \mathrm{mmr} .2013 .1385$

\begin{abstract}
Hepatic stellate cells (HSCs) are important in the development of liver fibrosis and in the pathogenesis of portal hypertension. Octreotide, an analogue of somatostatin, has been demonstrated to effectively treat fibrosis and portal hypertension; however, its relative mechanism in HSCs remains unknown. LX-2, the immortalized HSC line, was used to study the mechanism whereby octreotide functions at different concentrations. Real-time polymerase chain reaction (PCR) and western blot analysis were used to analyze the expression of fibrosis markers and transcription factors following treatment with octreotide. Soluble secreted endothelin-1 (ET-1), collagen I and vascular endothelial growth factor (VEGF) were assessed in the supernatants of cultured cells by enzyme-linked immunosorbent assay (ELISA). In the present study, it was shown that octreotide was able to inhibit the proliferative ability of the LX-2 cells and decrease the expression of transforming growth factor $\beta$ (TGF- $\beta), \alpha$-smooth muscle actin ( $\alpha$-SMA) and smad-4a. The transcription factors, including c-Jun and sp-1, were downregulated in a dose-dependent manner following treatment with octreotide. The levels of ET-1 and collagen I in the supernatant decreased significantly in contrast with the normal levels, whereas the levels of VEGF in the LX-2 cells and the supernatant increased at a high octreotide concentration $\left(10^{-5} \mathrm{nM}\right)$. Octreotide may exert its effects on ET-1 or other targeting genes in HSCs through the downregulation of c-Jun and specificity protein 1 (sp-1), and the increased levels of VEGF may be the reason for the side effects observed at high concentrations of octreotide.
\end{abstract}

Correspondence to: Professor Bo Han, Department of Pathology, Shandong University Medical School, 44 Wenhua Xi Road, Jinan, Shandong 250012, P.R. China

E-mail: author@mail.com

${ }^{*}$ Contributed equally

Key words: hepatic stellate cell, fibrosis, transcription factor, vascular endothelial growth factor

\section{Introduction}

Hepatic fibrosis is a healing response to all causes of chronic hepatic injury. However, it also leads to numerous clinically significant problems that are correlated with the progression of portal hypertension and liver failure $(1,2)$.

Hepatic stellate cells (HSCs) are a key source of extracellular matrix (ECM). The activation of HSCs is one of the key steps in the development of liver fibrosis $(3,4)$, which is a common pathological change characterized by an excessive deposition of ECM that occurs in the majority of types of chronic liver diseases. In vitro and in vivo studies have suggested that HSCs are also involved in the regulation of the liver microcirculation and the pathogenesis of portal hypertension (5). HSCs comprise $15 \%$ of the total number of resident liver cells and reside in the space of Disse in close contact with sinusoidal endothelial cells and hepatocytes (6). HSCs activated by liver injury lose retinoids and increase the level of ECM. HSCs also express $\alpha$-smooth muscle actin ( $\alpha$-SMA) and possess contractile abilities (7).

Somatostatin, a significant peptide for inhibiting cell proliferation and differentiation, is able to retard the growth of various types of cells by blocking the synthesis and/or secretion of numerous key cytokines and hormones (8-12). At a low dose, somatostatin may exert antiproliferative and proapoptotic activities on activated HSCs. This result may provide a basis for utilizing somatostatin and potentially its analogue, octreotide, to protect and treat individuals suffering from hepatic fibrosis. However, the underlying mechanisms require further investigation.

The primary objective of the present study was to evaluate the effects of octreotide on hepatic fibrosis in the immortalized HSC cell line, LX-2. The mechanism of octreotide in LX-2 was also investigated.

\section{Materials and methods}

Cell culture. The human immortalized HSC line, LX-2, was obtained from the Shanghai Fuxing Biotechnology Co., China. Cells were maintained in Dulbecco's modified Eagle's medium (DMEM) supplemented with $10 \%$ fetal bovine serum (FBS), $100 \mathrm{U} / \mathrm{ml}$ penicillin and $100 \mathrm{~g} / \mathrm{ml}$ streptomycin, and 
incubated in a $37^{\circ} \mathrm{C}$ humidified atmosphere with $5 \% \mathrm{CO}_{2}$. The cultured cells were then used in the subsequent experiments. The study was approved by the ethics committee of the Medical School of Shandong University, Jinan, China.

mRNA expression of specificity protein 1 (sp-1), c-Jun, $\alpha$-SMA, smad-4, vascular endothelial growth factor (VEGF) and transforming growth factor $\beta$ (TGF- $\beta$ ) in cultured cells. Cultured cells were homogenized in TRIzol (Invitrogen Life Technologies, Carlsbad, CA, USA) and centrifuged at $10,000 \mathrm{x}$ g, at $4^{\circ} \mathrm{C}$ for $15 \mathrm{~min}$, in the presence of chloroform. The upper aqueous phase was collected, and total RNA was precipitated by the addition of isopropanol and centrifugation at $7,500 \mathrm{x} \mathrm{g}$ at $4^{\circ} \mathrm{C}$ for $5 \mathrm{~min}$. RNA pellets were washed with $75 \%$ ethanol, dried and reconstituted with sterile water. The concentration was determined by measuring the absorbance at $490 \mathrm{~nm}$ with a spectrophotometer.

Total RNA was reverse transcribed in a final volume of $10 \mu 1$ using an RNA polymerase chain reaction (PCR) kit (Takara Bio, Inc., Katsushika, Tokyo, Japan). Primers were designed using Primer 5.0 software and the sequences were as follows: Human c-Jun forward, 5'-AGGAGGAGCCTC AGACAGTG-3' and reverse, 5'-TGTTTAAGCTGTGCC ACCTG-3'; $\alpha$-SMA forward, 5'-AGGGAGTAATGGTTG GAATGGG-3' and reverse, 5'-GGAGTACGGTACGCAGA-3'; smad-4 forward, 5'-GGCAGCCATAGTGAAGGACTG-3' and reverse, 5'-GGC GGGTGGTGCTGAAGATGG-3'; sp-1 forward, 5'-AAG AAATGACCTTAGGAACATACCC-3' and reverse, 5'-CCGTATATGTCTACACACAGATGAC-3'; VEGF forward, 5'-CGGGAACCAGATCTCTCACC-3' and reverse, 5'-AAAATGGCGAATCCAATTCC-3'; TGF- $\beta$ forward, 5'-AAGGCCAAATATCCCAAACA-3' and reverse, 5'-CCA ACATTCTCTCATAATTTTAGCC-3'; glyceraldehyde 3-phosphate dehydrogenase (GAPDH; used as an endogenous standard) forward, 5'-ACATGTTCCAATATGATTCC-3' and reverse, 5'-TGGACTCCACGACGTACTCAG-3'. Real-time PCR reactions were conducted using the LightCycler 480 instrument (Roche Molecular Biochemicals, Mannheim, Germany) and performed according to the manufacturer's instructions. Reactions were conducted in a tube with a total volume of $10 \mu \mathrm{l}$, which consisted of $1 \mu \mathrm{l}$ cDNA, $5 \mu \mathrm{l}$ SYBR-Green real-time PCR master mix (Toyobo Co., Ltd., Osaka, Japan) and $1 \mu \mathrm{l}$ each primer. Fluorescent signals were captured during each of the 40 cycles (denaturizing for $10 \mathrm{sec}$ at $95^{\circ} \mathrm{C}$, annealing for $15 \mathrm{sec}$ at $60^{\circ} \mathrm{C}$ and extension for $30 \mathrm{sec}$ at $72^{\circ} \mathrm{C}$ ). GAPDH was used as a reference gene for normalization and water was used as the negative control. Relative quantification was calculated using the comparative threshold cycle (CT), which was inversely related to the abundance of mRNA transcripts in the initial sample. The mean $\mathrm{CT}$ of the duplicate measurements was used to calculate $\Delta \mathrm{CT}$ as the difference in CT for the target and reference. The relative quantity of the product was expressed as the fold-induction of the target gene compared with the reference gene, according to the formula $2^{-\Delta \Delta C T}$, where $\Delta \Delta \mathrm{CT}$ represented $\Delta \mathrm{CT}$ values normalized with the mean $\Delta \mathrm{CT}$ of the control samples.

Western blot analysis of c-Jun, sp-1,VEGF and GAPDH in cultured cells. Cultured cells were individually homogenized in a buffer solution with $20 \mathrm{~mm}$ Tris- $\mathrm{HCl}(\mathrm{pH}$ 7.4) containing 1\% Triton X-100, 0.1\% SDS, $50 \mathrm{~mm} \mathrm{NaCl}, 2.5 \mathrm{~mm}$ EDTA,
$1 \mathrm{~mm} \mathrm{Na}{ }_{4} \mathrm{P}_{2} \mathrm{O}_{7}, 10 \mathrm{~mm} \mathrm{H}_{2} \mathrm{O}, 20 \mathrm{~mm} \mathrm{NaF}, 1 \mathrm{~mm} \mathrm{Na}_{3} \mathrm{VO}_{4}$, $2 \mathrm{~mm}$ Pefabloc and a cocktail of protease inhibitors (Complete Mini; Roche Applied Science, Indianapolis, IN, USA), then centrifuged at $16,000 \mathrm{x}$ g for $5 \mathrm{~min}$ at $4^{\circ} \mathrm{C}$. Supernatants were collected following centrifugation, and the protein concentrations were determined using the BCA Protein Assay kit (Thermo Fisher Scientific, Rockford, IL, USA). Total protein was separated by sodium dodecyl sulphate polyacrylamide gel electrophoresis (SDS-PAGE) and transblotted onto nitrocellulose membranes (GE Healthcare, Piscataway, NJ, USA). Western blot analysis was conducted using antibodies against c-Jun, sp-1 and VEGF (Abcam, Cambridge,UK) at a 1,000-fold dilution. All primary and secondary antibodies were diluted in 5\% dry skimmed milk in Tris-buffered saline with Tween [TBST; $0.02 \mathrm{M}$ Tris base and $0.137 \mathrm{M} \mathrm{NaCl}$ in distilled water (pH 7.6) containing $0.1 \%$ Tween-20]. Immunosignals were visualized with the Protein Detector BCIP/NBT Western Blot kit (Beyotime Institute of Biotechnology, Shanghai, China) according to the manufacturer's instructions. Quantification was conducted using ImageQuant 5.2 software. An additional membrane prepared following the same protocol was probed with anti-GADPH antibodies (Santa Cruz Biotechnology, Inc., Santa Cruz, CA, USA) to normalize the sample loading.

MTT assay. LX-2 was plated into 96-well plates at a density of $5 \times 10^{5}$ cells/well in $100 \mu \mathrm{M}$ growth medium, and allowed to grow overnight to reach $\sim 85 \%$ confluence. Three different concentrations of octreotide in $100 \mathrm{ml}$ growth medium $\left(10^{-5}, 10^{-6}\right.$ and $10^{-7} \mathrm{nM}$ ) were added to the wells. Following incubation for $48 \mathrm{~h}$, the 3-(4,5-dimethylthiazol-2-yl)-2,5-diphenyltetrazolium bromide (MTT) assay was used to measure cell proliferation. In brief, $20 \mathrm{ml}$ of $5 \mathrm{mg} / \mathrm{ml}$ MTT was added to each well and the cells were incubated at $37^{\circ} \mathrm{C}$ for $4 \mathrm{~h}$. The MTT was then removed and replaced with $100 \mathrm{ml}$ dimethyl sulfoxide (Sigma, St. Louis, MO, USA) for further incubation for $10 \mathrm{~min}$ at $37^{\circ} \mathrm{C}$, until the crystals had dissolved. The optical density (OD) value of each well was measured using a microplate reader (ELx800; BioTek Instruments, Winooski, VT, USA) with a test wavelength of $570 \mathrm{~nm}$. For each concentration or control, triplicate determinations were made.

Measurement of soluble secreted ET-1, collagen I and $V E G F$. Cells were seeded in high-glucose Dulbecco's Modified Eagle's Medium (DMEM) containing 1\% FBS $24 \mathrm{~h}$ prior to octreotide treatment. The total soluble secreted ET-1 (R\&D Systems, Minneapolis, MN, USA), VEGF (R\&D Systems) and collagen I (Cosmo Bio, Co., Ltd., Tokyo, Japan) in the culture supernatants in the absence or presence of octreotide for $48 \mathrm{~h}$ was measured using a sandwich ELISA, according to the manufacturer's instructions for each kit. Briefly, microtiter wells were pre-coated for overnight 1:20 dilutions of LX-2 cell conditioned medium supernatant. The plates were then developed by the addition of biotinylated antibodies against ET-1, VEGF or collagen I, followed by avidin-conjugated horseradish peroxidase. The color reaction was developed using a tetramethylbenzidine substrate solution and the absorbance was measured at $450 \mathrm{~nm}$ using a Model 550 plate reader (BioRad, Hercules, CA, USA). The assay was performed in triplicate and the mean values of each sample were calculated. 
Statistical analysis. The statistical significance of the differences between the means was assessed by a one-way analysis of variance (ANOVA) for multiple comparisons and an independent-sample Student's t-test. Differences between two or more groups were analyzed using the Mann-Whitney U test or the Kruskal-Wallis test. Statistical analyses were performed with SPSS 13.0 (SPSS, Inc., Chicago, IL, USA). P $<0.05$ was considered to indicate a statistically significant difference.

\section{Results}

Octreotide inhibits $L X-2$ proliferation. To investigate the function of the analogue of somatostatin, octreotide, we analyzed the rate of cell growth in LX-2 cells following treatment at concentrations of $10^{-5}, 10^{-6}$ and $10^{-7} \mathrm{nM}$. LX-2 cells without any treatment were selected as the controls. The results showed a significant decrease in cell proliferation, in a concentration- dependent manner, compared with the controls, when examined by the MTT assay $(\mathrm{P}<0.05)$. In addition, the inhibitory rates of the octreotide treatment groups increased to 27.5 and $49.8 \%$ when the concentration was $10^{-7}$ and $10^{-5} \mathrm{nM}$, respectively (Fig. 1).

Octreotide alleviates the fibrogenesis function of HSCs. In order to determine whether octreotide was able to alleviate fibrosis in LX- 2 cells, we selected TGF- $\beta$ (a potent profibrogenic cytokine proposed to play a central role in regulating tissue fibrosis), $\alpha$-SMA and smad- $4 \alpha$ (two major profibrogenic cytokines implicated in the development of liver fibrosis). Real-time PCR analysis of extracted mRNA samples of the cultured cells revealed decreased levels of TGF- $\beta 1$ (Fig. 2A), $\alpha$-SMA (Fig. 2B) and smad- $4 \alpha$ (Fig. 2C) expression in the octreotide group, thus illustrating its inhibitory effects on profibrogenic gene expression.

ET-1 and collagen I expression levels decrease following treatment with octreotide. To investigate whether octreotide was directly involved in collagen I and ET-1 production in activated HSCs, we analyzed the effect of octreotide on secreted collagen I and ET-1 production using the LX-2 cell line. As indicated, the level of soluble secreted ET-1 (Fig. 3A) and collagen I (Fig. 3B) in the supernatant of the octreotide-treated LX-2 cells was lower than that produced by the control cells.

Octreotide attenuates hypertension and fibrosis through the inhibition of c-Jun and sp-1 expression. Previous studies have demonstrated that IFN- $\gamma$ signals to c-Jun to negatively regulate preproET-1 transcription. Combined with our results, it may be proposed that octreotide decreases ET-1 expression. We hypothesized that octreotide may target c-Jun or other transcription factors, either directly or indirectly, which in turn may mediate the inhibitory effect of octreotide on the expression of fibrosis-related genes. Sp-1 also demonstrated a protective function against apoptosis and injury. As demonstrated in Fig. 4A, the c-Jun protein levels significantly decreased compared with the control, following octreotide exposure for $48 \mathrm{~h}$ (Fig. 4A). We also examined c-Jun mRNA expression following octreotide treatment. Notably, the c-Jun protein levels were markedly reduced at $48 \mathrm{~h}$, while octreotide maintained its inhibitory effect throughout this time (Fig. 4C).

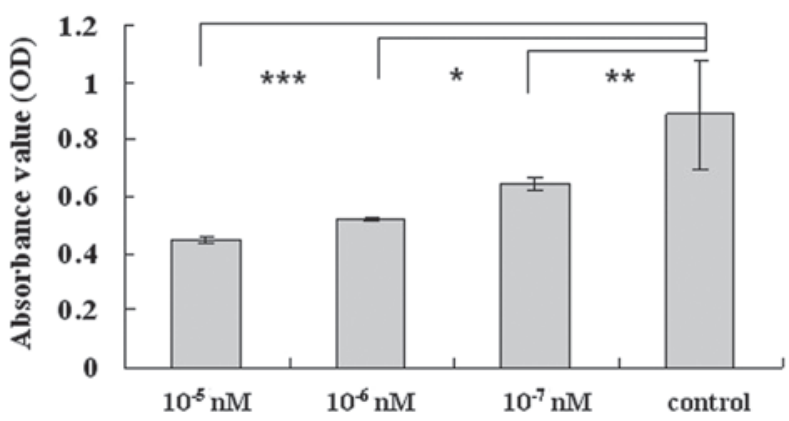

Figure 1. Effect of octreotide on the proliferation of LX-2 cells. Octreotide inhibits the proliferation of LX-2 cells in a dose-dependent manner, as demonstrated by the 3-(4,5-dimethylthiazol-2-yl)-2,5-diphenyltetrazolium bromide (MTT) assay. ${ }^{*} \mathrm{P}<0.05,{ }^{* *} \mathrm{P}<0.01$ and ${ }^{* * *} \mathrm{P}<0.001$. OD, optical density.

In addition, the level of sp-1 was observed to decrease at the transcriptional and translational levels (Fig. 4B and D, respectively). In conclusion, octreotide may ameliorate fibrosis by downregulating c-Jun and sp-1.

Octreotide increases VEGF expression in LX-2 cells. To analyze the effects of octreotide on VEGF activity, LX-2 cells were treated with octreotide for $48 \mathrm{~h}$ at various concentrations. The VEGF mRNA transcription, protein and secreted soluble protein levels were quantified by real-time RT-PCR, western blot analysis and ELISA, respectively. Octreotide increased the expression of VEGF mRNA when at a higher concentration $\left(10^{-5} \mathrm{nM}\right)$, but this declined to the normal level as the concentration was decreased. The ELISA results revealed the same tendency for VEGF at a high octreotide concentration $\left(10^{-5} \mathrm{nM}\right.$; Fig. 5A-C).

\section{Discussion}

Activated hepatic stellate cells (HSCs) are key participants in hepatic fibrosis. In vivo and in vitro studies have suggested that HSCs are also involved in the regulation of the liver microcirculation and the pathogenesis of portal hypertension. Thus, the induction of HSC apoptosis has been proposed as an antifibrotic treatment strategy. Consistent with other studies (13), at a low dose, octreotide, the analogue of somatostatin, was demonstrated to exert antiproliferative actions on LX-2 cells and to inhibit the expression of $\alpha$-SMA, smad- 4 and TGF- $\beta$, which are all markers of fibrosis. The aforementioned LX-2 cell line makes it feasible to clarify the mechanism whereby octreotide exerts its effects on HSCs.

ET-1, a powerful vasoconstrictor peptide, is produced by activated HSCs and promotes cell proliferation, fibrogenesis and contraction; the latter of which has been proposed to be mechanistically linked to portal hypertension in cirrhosis. In the present study, we found that octreotide downregulated ET-1 expression in LX-2 cells, which suggested an additional plausible pathway that ET-1 may use during the attenuation of portal hypertension. In a study by $\mathrm{Li}$ et al, it was demonstrated that the IFN $\gamma$-induced inhibition of preproET-1 mRNA expression was closely linked to the AP-1 and Smad3 signaling pathways (14). IFN $\gamma$ reduced JNK phosphorylation, which was correlated with the decrease in phosphorylation of downstream factors, c-Jun 
A

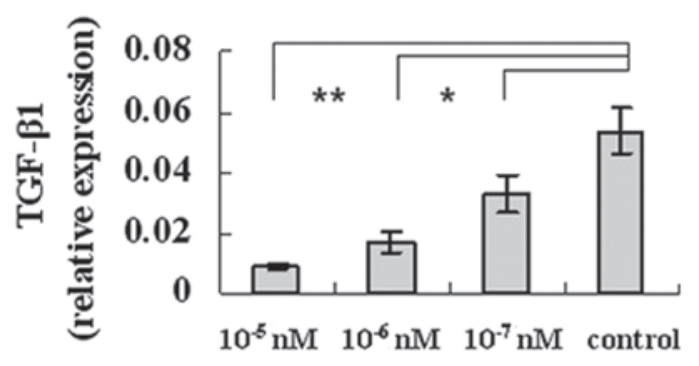

B

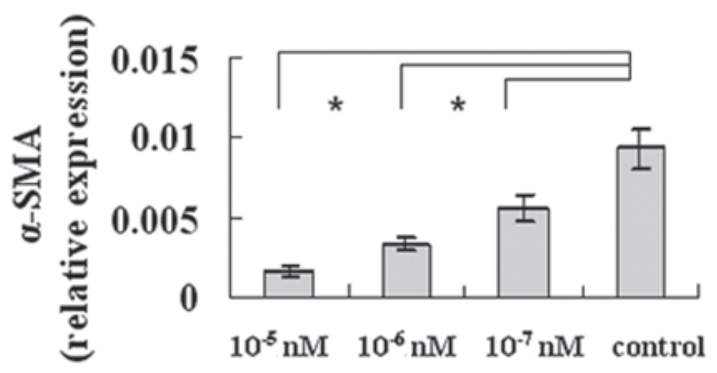

C

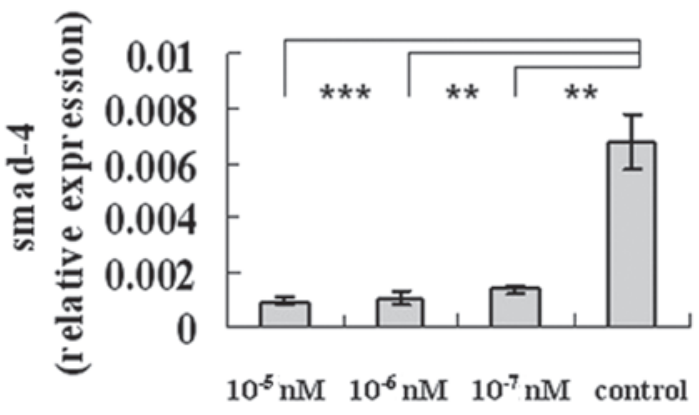

Figure 2. Relative transforming growth factor $\beta$ (TGF- $\beta 1$ ), $\alpha$-smooth muscle actin ( $\alpha$-SMA) and smad-4 expression in LX-2 cells. The mRNA expression of (A) TGF- $\beta 1$, (B) $\alpha$-SMA and (C) smad- 4 was studied by relative quantitative real-time RT-PCR in LX- 2 cells exposed to various concentrations of octreotide. The results were corrected for glyceraldehyde 3 -phosphate dehydrogenase (GAPDH) mRNA expression within each sample. ${ }^{*} \mathrm{P}<0.05,{ }^{* *} \mathrm{P}<0.01$ and ${ }^{* * *} \mathrm{P}<0.001$. Results are the average of three separate experiments performed in triplicate, with bars representing the standard error.

A

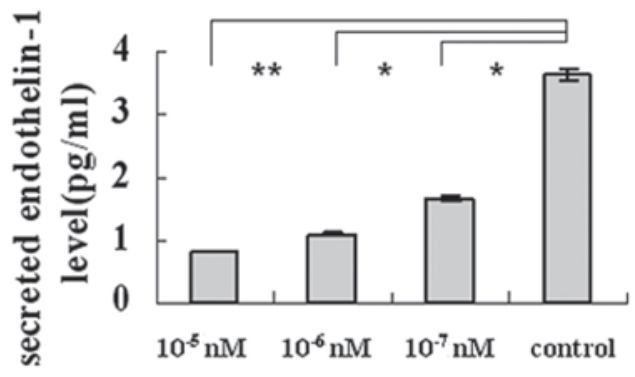

B

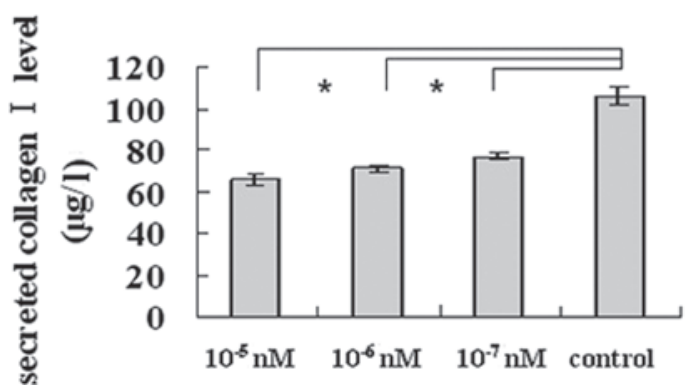

Figure 3. Effect of octreotide on secretion of endothelin-1 (ET-1) or collagen I. The figure shows the protein levels of (A) ET-1 and (B) collagen I in conditioned medium measured by enzyme-linked immunosorbent assay (ELISA) following treatment of the cells with octreotide. ${ }^{*} \mathrm{P}<0.05,{ }^{* *} \mathrm{P}<0.01$ and ${ }^{* * *} \mathrm{P}<0.001$.

and Smad3, and the decrease in the binding activity of c-Jun and Smad3 in the preproET-1 promoter. Notably, IFN- $\gamma$ reduced c-Jun mRNA and protein levels (14). To investigate whether transcription factors participated in octreotide-induced effects, we screened susceptible factors, including cox-2, c-fos, c-Jun, sp-1, c-myc, fra-1, vhl and Jun-B, and found that the c-Jun and sp-1 levels significantly decreased (the data with regard to the remaining transcription factors are not shown), suggesting that the two transcription factors may be accountable for the effects of octreotide.

It has been demonstrated that c-Jun is able to promote pulmonary artery endothelial cell (PAEC) growth and angiogenesis during pulmonary arterial hypertension (15). Furthermore, c-Jun also functions as a downstream mediator of the pro-fibrotic effects of TGF- $\beta$ and platelet-derived growth factor (PDGF) in systemic sclerosis (SSc) fibroblasts (16). Drugs that block the p38/AP-1 pathway may inhibit liver extracellular matrix synthesis and suppress liver fibrosis (17). Certain studies have found that all-trans retinoic acid (ATRA) was capable of inhibiting the proliferation and collagen production of HSCs via the suppression of active protein-1 and the c-Jun N-terminal kinase signal, and subsequently by decreasing the mRNA expression of the profibrogenic genes (18-20). The aforementioned findings suggest that c-Jun has important roles in the octreotide-induced ease of portal hypertension and in decreased fibrosis.

An increased activation of sp-1 was identified under conditions in which estrodial enhanced growth and reduced $\mathrm{TNF} \alpha$-induced apoptosis in human umbilical vein endothelial cells (HUVEC) (21). Evans et al demonstrated that signaling through CD31 in endothelial cells leads to protection from apoptosis in association with activation of the transcription factor sp-1 (22). Kang and Chen demonstrated that curcumin inhibits srebp-2 expression in cultured HSCs by reducing sp-1 activity (23). Furthermore, the transient overexpression of integrin $\alpha v \beta 5$ in normal fibroblasts has been demonstrated to enhance human $\alpha 2$ (I) collagen promoter activity through 
A

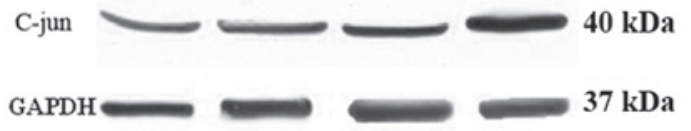

B

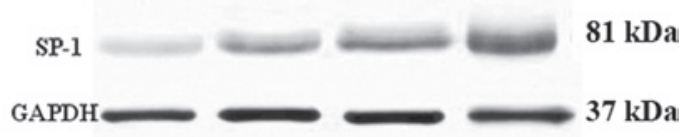

C

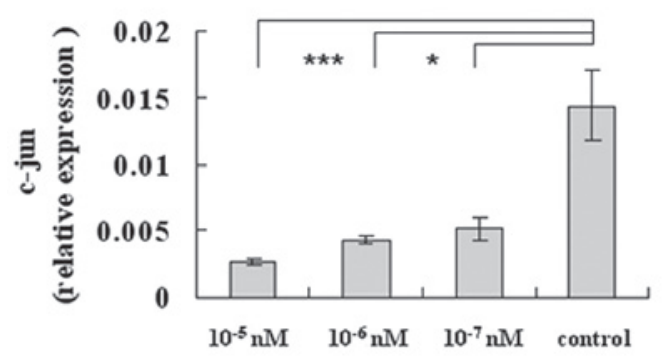

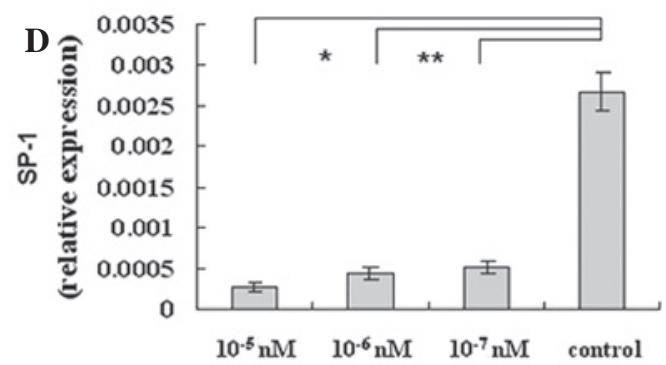

Figure 4. Effect of octreotide treatment on c-Jun and specificity protein 1 (sp-1) in LX-2 cells. (A) LX-2 cells were treated with or without octreotide at varying concentrations. Protein extracts of the cells were investigated by western blot analysis for (A) c-Jun and (B) sp-1 expression. Glyceraldehyde 3-phosphate dehydrogenase (GAPDH) was used as the loading control. ${ }^{*} \mathrm{P}<0.05,{ }^{* *} \mathrm{P}<0.01$ and ${ }^{* * * *} \mathrm{P}<0.001$. The transcriptional levels of $(\mathrm{C}) \mathrm{c}-\mathrm{Jun}$ and $(\mathrm{D})$ sp-1 were detected using real-time PCR.

A

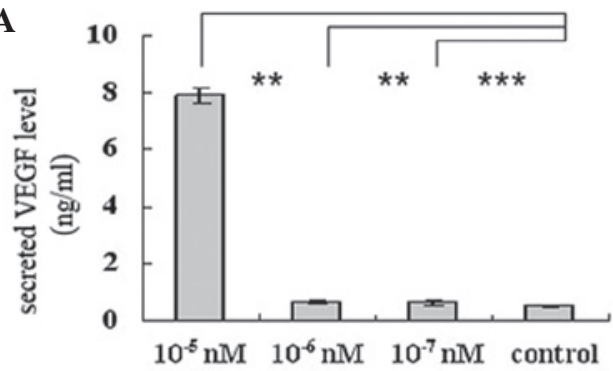

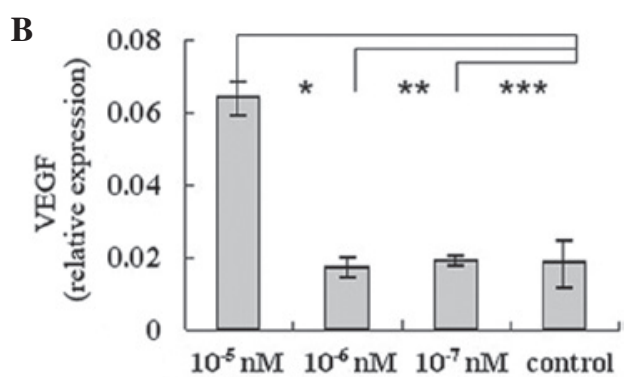

C

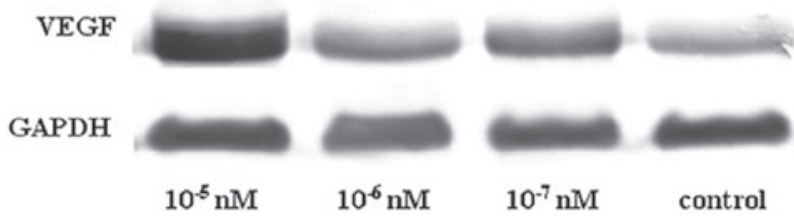

$43 \mathrm{kDa}$

37 kDa

Figure 5. In vivo assessment of vascular endothelial growth factor (VEGF) expression in LX-2 cells under octreotide stimulation. (A) The serum levels of VEGF in the LX-2 cell supernatants were analyzed $48 \mathrm{~h}$ following treatment with octreotide. ${ }^{* * *} \mathrm{P}<0.001$ and ${ }^{* *} \mathrm{P}<0.01$. (B) The expression of VEGF mRNA in LX-2 cells stimulated with octreotide quantified using real-time RT-PCR. The mRNA level was normalized relative to the level of the transcript of glyceraldehyde 3-phosphate dehydrogenase (GAPDH), a housekeeping gene. An unpaired Student's t-test was used for the statistical analysis. ${ }^{* * *} \mathrm{P}<0.001,{ }^{* *} \mathrm{P}<0.01$ and ${ }^{*} \mathrm{P}<0.05$. (C) Western blot analysis of VEGF $48 \mathrm{~h}$ following treatment with octreotide. The expression was analyzed using anti-VEGF antibodies.

sp-1 (24). Although the targeting genes of c-Jun and sp-1 require further study, the fact that these genes are involved in octreotide-induced effects suggests that it may be possible to develop medicines that target these transcription factors, in order to treat fibrosis and portal hypertension in the liver.

In the present study, we also found that the expression of VEGF increased with $10^{-5} \mathrm{nM}$ octreotide, but decreased to normal levels when the octreotide concentration declined. As determined previously, VEGF promoted angiogenesis and fibrosis, which affect portal hypertention, under abnormal conditions. However, the in vitro antiangiogenic effects of somatostatin and its analogues are likely to be efficiently counterbalanced in the tumor microenvironment by the concomitant release of proangiogenic factors, including VEGF (25), which may account for the elevated level of VEGF in our study. Experimental and clinical studies have clearly demonstrated that hepatic angiogenesis, irrespective of aetiology, occurs in conditions of chronic liver diseases (CLDs), and is characterized by perpetuation of cell injury and death, the inflammatory response and progressive fibrogenesis. Angiogenesis and associated changes in the liver vascular architecture, which in turn increase vascular resistance and portal hypertension and decrease parenchymal perfusion, have been proposed to favour the fibrogenic progression of the disease towards the endpoint of cirrhosis. Therefore, upregulated VEGF may be accountable for the side-effects of octreotide treatment at high doses (26). Rosmorduc demonstrated that antiangiogenic therapies may therefore, by limiting liver fibrosis and 
inflammation in cirrhosis, prevent the occurrence of severe complications, including portal hypertension and potentially, liver cancer (27). Therefore, the combination of octreotide and antiangiogenic therapy has potential if long-term administration at a high concentration is necessary.

There were certain limitations to the present study; the transcription factors were not screened systematically and their exact roles remained unknown. Therefore, further study is required.

In the present study, we have demonstrated that the effects of octreotide on HSCs were elicited to alleviate fibrosis and hypertension by downregulating c-Jun and sp-1, and that the downstream targets require further investigation. Furthermore, VEGF levels increased at a high octreotide concentration, which may explain the side-effects of a long-term administration of octreotide at high concentrations.

\section{Acknowledgements}

This study was supported by a grant from the Shandong Science and Technology Research Program (grant no. 2010G0020223).

\section{References}

1. Arroyo $\mathrm{V}$, Terra $\mathrm{C}$ and Ginès $\mathrm{P}$ : Advances in the pathogenesis and treatment of type-1 and type-2 hepatorenal syndrome. J Hepatol 46: 935-946, 2007.

2. Friedman SL: Liver fibrosis - from bench to bedside. J Hepatol 38 (Suppl 1): S38-S53, 2003.

3. Bartlett ST, Kuo PC, Johnson LB, Lim JW and Schweitzer EJ: Pancreas transplantation at the University of Maryland. Clin Transpl: 271-280, 1996.

4. Sutherland DE, Gruessner R, Gillingham K, et al: A single institution's experience with solitary pancreas transplantation: a multivariate analysis of factors leading to improved outcome. Clin Transpl: 141-152, 1991.

5. Watts SW, Yang P, Banes AK and Baez M: Activation of Erk mitogen-activated protein kinase proteins by vascular serotonin receptors. J Cardiovasc Pharmacol 38: 539-551, 2001.

6. Li T, Weng SG, Leng XS, et al: Effects of 5-hydroxytamine and its antagonists on hepatic stellate cells. Hepatobiliary Pancreat Dis Int 5: 96-100, 2006.

7. Friedman SL: Molecular regulation of hepatic fibrosis, an integrated cellular response to tissue injury. J Biol Chem 275: 2247-2250, 2000.

8. Xia D, Zhao RQ, Wei XH, Xu QF and Chen J: Developmental patterns of $\mathrm{GHr}$ and SS mRNA expression in porcine gastric tissue. World J Gastroenterol 9: 1058-1062, 2003.

9. Vindeløv SD, Hartoft-Nielsen ML, Rasmussen AK, et al: Interleukin-8 production from human somatotroph adenoma cells is stimulated by interleukin- $1 \beta$ and inhibited by growth hormone releasing hormone and somatostatin. Growth Horm IGF Res 21: 134-139, 2011.

10. Yao YL, Xu B, Zhang WD and Song YG: Gastrin, somatostatin, and experimental disturbance of the gastrointestinal tract in rats. World J Gastroenterol 7: 399-402, 2001.
11. Sun FP, Song YG, Cheng W, Zhao T and Yao YL: Gastrin somatostatin, G and D cells of gastric ulcer in rats. World J Gastroenterol 8: 375-378, 2002.

12. Li YY: Mechanisms for regulation of gastrin and somatostatin release from isolated rat stomach during gastric distention. World J Gastroenterol 9: 129-133, 2003.

13. Reynaert H, Thompson MG, Thomas T and Geerts A: Hepatic stellate cells: role in microcirculation and pathophysiology of portal hypertension. Gut 50: 571-581, 2002.

14. Li T, Shi Z and Rockey DC: Preproendothelin-1 expression is negatively regulated by IFN $\gamma$ during hepatic stellate cell activation. Am J Physiol Gastrointest Liver Physiol 302: G948-G957, 2012.

15. Ma J, Zhang L, Han W, et al: Activation of JNK/c-Jun is required for the proliferation, survival, and angiogenesis induced by EET in pulmonary artery endothelial cells. J Lipid Res 53: 1093-1105, 2012.

16. Fuest M, Willim K, MacNelly S, et al: The transcription factor c-Jun protects against sustained hepatic endoplasmic reticulum stress thereby promoting hepatocyte survival. Hepatology 55 : 408-418, 2012.

17. Reich N, Tomcik M, Zerr P, et al: Jun N-terminal kinase as a potential molecular target for prevention and treatment of dermal fibrosis. Ann Rheum Dis 71: 737-745, 2012.

18. Zhang Y and Yao X: Role of c-Jun N-terminal kinase and p38/activation protein-1 in interleukin-1 $\beta$-mediated type I collagen synthesis in rat hepatic stellate cells. APMIS 120: 101-107, 2012.

19. Avouac J, Palumbo K, Tomcik M, et al: Inhibition of activator protein 1 signaling abrogates transforming growth factor $\beta$-mediated activation of fibroblasts and prevents experimental fibrosis. Arthritis Rheum 64: 1642-1652, 2012.

20. Ye Y and Dan Z: All-trans retinoic acid diminishes collagen production in a hepatic stellate cell line via suppression of active protein-1 and c-Jun N-terminal kinase signal. J Huazhong Univ Sci Technolog Med Sci 30: 726-733, 2010.

21. Ling S, Zhou L, Li H, et al: Effects of 17beta-estradiol on growth and apoptosis in human vascular endothelial cells: influence of mechanical strain and tumor necrosis factor-alpha. Steroids 71: 799-808, 2006

22. Evans PC, Taylor ER and Kilshaw PJ: Signaling through CD31 protects endothelial cells from apoptosis. Transplantation 71: 457-460, 2001.

23. Kang Q and Chen A: Curcumin inhibits srebp-2 expression in activated hepatic stellate cells in vitro by reducing the activity of specificity protein-1. Endocrinology 150: 5384-5394, 2009.

24. Asano Y, Ihn H, Yamane K, Kubo M and Tamaki K: Increased expression levels of integrin alphavbeta5 on scleroderma fibroblasts. Am J Pathol 164: 1275-1292, 2004.

25. Walter T, Hommell-Fontaine J, Gouysse G, et al: Effects of somatostatin and octreotide on the interactions between neoplastic gastroenteropancreatic endocrine cells and endothelial cells: a comparison between in vitro and in vivo properties. Neuroendocrinology 94: 200-208, 2011.

26. Paternostro C, David E, Novo E and Parola M: Hypoxia, angiogenesis and liver fibrogenesis in the progression of chronic liver diseases. World J Gastroenterol 16: 281-288, 2010.

27. Rosmorduc O: Antiangiogenic therapies in portal hypertension: a breakthrough in hepatology. Gastroenterol Clin Biol 34: 446-449, 2010. 